\title{
Ramanujan's evaluations of Rogers-Ramanujan type continued fractions at primitive roots of unity
}

by

\author{
Sen-Shan Huang (Urbana, Ill.)
}

\section{Introduction. Let}

$$
\mathbb{K}\left(a_{n} / 1\right):=\frac{a_{1}}{1}+\frac{a_{2}}{1}+\frac{a_{3}}{1}+\ldots
$$

be a continued fraction and let

$$
\frac{P_{n}}{Q_{n}}:=\frac{a_{1}}{1}+\frac{a_{2}}{1}+\ldots+\frac{a_{n}}{1}, \quad n \geq 1,
$$

be the $n$th convergent (or approximant) of $\mathbb{K}\left(a_{n} / 1\right)$. Set $P_{-1}=1, Q_{-1}=0$, $P_{0}=0$, and $Q_{0}=1$. By convention, the value of $\mathbb{K}\left(a_{n} / 1\right)$, if it exists, is defined to be the limit of the sequence $\left\{P_{n} / Q_{n}\right\}$ as $n$ tends to infinity.

Some basic and important facts about $P_{n}$ and $Q_{n}[2$, p. 9] are

$$
P_{n}=P_{n-1}+a_{n} P_{n-2}, \quad Q_{n}=Q_{n-1}+a_{n} Q_{n-2},
$$

and

$$
P_{n} Q_{n-1}-P_{n-1} Q_{n}=(-1)^{n-1} a_{1} \ldots a_{n},
$$

where $n=1,2, \ldots$

Now, by putting $a_{n}=a x^{n-1}$ in (1.1), we obtain the continued fraction

$$
R(a):=\frac{a}{1}+\frac{a x}{1}+\frac{a x^{2}}{1}+\ldots
$$

On page 46 of his lost notebook [5], Ramanujan evaluates $R(a)$ in terms of its $(m-1)$ th convergent when $x$ is a primitive $m$ th root of unity. More precisely, Ramanujan claims that, given any primitive $m$ th root of unity $x$,

$$
R(a)=\frac{P(a)+\frac{1}{2}\left(-1+\sqrt{1+4 a^{m}}\right)}{Q(a)},
$$

1991 Mathematics Subject Classification: Primary 11A55; Secondary 40A15, 11P83.

Key words and phrases: continued fraction, convergent, primitive root of unity, partition modulo $s$. 
where

$$
\frac{P(a)}{Q(a)}=\frac{a}{1}+\frac{a x}{1}+\ldots+\frac{a x^{m-2}}{1}
$$

is the $(m-1)$ th convergent of $R(a)$. Note that one can obtain this kind of result simply by solving a quadratic equation. However, to prove (1.6) requires more than that. Especially, nontrivial observations are needed.

In Section 2, we proceed with the study of the convergents of $R(a)$. In particular, Theorem 2.2 is one of the main ingredients needed to prove (1.6). It turns out that Theorem 2.2 can be established by proving an interesting identity (2.1).

The third section is devoted to the proof of (2.1). Arising naturally in the proof is the notion of relative decompositions $(\bmod m)$ which was first introduced and studied by Stern [8], [9] and then further developed and generalized by von Sterneck [10]-[12].

In Section 4, (1.6) is restated as Theorem 4.4 in a more precise way and is proved by using results of Worpitzky (Lemma 4.1) and Vitali (Lemma 4.2).

In the final section, we generalize Theorem 4.4 by proving some results on page 57 of Ramanujan's lost notebook.

2. On convergents of $R(a)$. To simplify notation, we define the two sets,

$\mathcal{A}_{n}:=\left\{\vec{v}=\left(n_{1}, \ldots, n_{r}\right) \in \mathbb{N}^{r}: r \geq 1, n_{1}=1, n_{i+1}-n_{i} \geq 2\right.$, and $\left.n_{r} \leq n\right\}$, and

$\mathcal{B}_{n}:=\left\{\vec{v}=\left(n_{1}, \ldots, n_{r}\right) \in \mathbb{N}^{r}: r \geq 1, n_{1} \geq 2, n_{i+1}-n_{i} \geq 2\right.$, and $\left.n_{r} \leq n\right\}$.

Lemma 2.1. For each positive integer $n$,

$$
P_{n}=\sum_{\vec{v} \in \mathcal{A}_{n}} a_{n_{1}} \ldots a_{n_{r}}
$$

and

$$
Q_{n}=1+\sum_{\vec{v} \in \mathcal{B}_{n}} a_{n_{1}} \ldots a_{n_{r}}
$$

where $P_{n}$ and $Q_{n}$ are defined in (1.2).

Pr o of of (i). Use induction on $n$. Clearly (i) is valid for $n=1$. Assume that (i) is true up to $n$. Then, by the first recurrence relation in (1.3), 


$$
\begin{aligned}
P_{n+1} & =P_{n}+a_{n+1} P_{n-1} \\
& =\sum_{\vec{v} \in \mathcal{A}_{n}} a_{n_{1}} \ldots a_{n_{r}}+\sum_{\vec{v} \in \mathcal{A}_{n-1}} a_{n_{1}} \ldots a_{n_{r}} a_{n+1} \\
& =\sum_{\vec{v} \in \mathcal{A}_{n+1}} a_{n_{1}} \ldots a_{n_{r}} .
\end{aligned}
$$

Identity (ii) can be proved in a similar manner by using the second recurrence relation in (1.3). i.e.,

In the sequel, we will denote the $n$th convergent of $R(a)$ by $P_{n}(a) / Q_{n}(a)$,

$$
\frac{P_{n}(a)}{Q_{n}(a)}=\frac{a}{1}+\frac{a x}{1}+\ldots+\frac{a x^{n-1}}{1} .
$$

THEOREM 2.2. For any number $a$, and any primitive $m$ th root of unity $x$,

$$
P_{m-1}(a)+Q_{m}(a)=1 \text {. }
$$

Before we prove Theorem 2.2, let us take a closer look at the sum of $P_{m-1}(a)$ and $Q_{m}(a)$. First, define $\mathcal{A}_{n}(l)$ to be the subset of $\mathcal{A}_{n}$ which contains all the $l$-dimensional vectors. Similarly, $\mathcal{B}_{n}(l)$ contains all the $l$ dimensional vectors in $\mathcal{B}_{n}$. Then, by Lemma 2.1, we find that

$$
\begin{aligned}
P_{m-1}(a)+Q_{m}(a) & =\sum_{\vec{v} \in \mathcal{A}_{m-1}} a x^{n_{1}-1} a x^{n_{2}-1} \ldots a x^{n_{r}-1}+1+\sum_{\vec{v} \in \mathcal{B}_{m}} a x^{n_{1}-1} a x^{n_{2}-1} \ldots a x^{n_{r}-1} \\
= & 1+\sum_{r=1}^{[m / 2]} a^{r} x^{-r} \sum_{\vec{v} \in \mathcal{A}_{m-1}(r)} x^{n_{1}+\ldots+n_{r}}+\sum_{r=1}^{[m / 2]} a^{r} x^{-r} \sum_{\vec{v} \in \mathcal{B}_{m}(r)} x^{n_{1}+\ldots+n_{r}} \\
= & 1+\sum_{r=1}^{[m / 2]} a^{r} x^{-r}\left(\sum_{\vec{v} \in \mathcal{A}_{m-1}(r)} x^{n_{1}+\ldots+n_{r}}+\sum_{\vec{v} \in \mathcal{B}_{m}(r)} x^{n_{1}+\ldots+n_{r}}\right) \\
= & 1+\sum_{r=1}^{[m / 2]} a^{r} x^{-r}\left(\sum_{\vec{v} \in \mathcal{C}_{m}(r)} x^{n_{1}+\ldots+n_{r}}\right),
\end{aligned}
$$

where $\mathcal{C}_{m}(r)$ is the union of $\mathcal{A}_{m-1}(r)$ and $\mathcal{B}_{m}(r)$.

Therefore, to prove Theorem 2.2, it suffices to show that, given any primitive $m$ th root of unity $x$,

$$
\sum_{\vec{v} \in \mathcal{C}_{m}(r)} x^{n_{1}+\ldots+n_{r}}=0, \quad \text { for each } r=1,2, \ldots,[m / 2] .
$$

We will prove (2.1) as a corollary of a result in the following section. 
3. Relative decompositions $(\bmod m)$. The notion of the following definition was first introduced by Stern [8] and the name relative decompositions $(\bmod m)$ was given by Bachmann [1, Part II, Chap. 5].

Definition. Let $n$ be a positive integer. A sequence $\left(n_{1}, \ldots, n_{r}\right)$ of positive integers is called a relative decomposition $(\bmod m)$ of $n$ (with $r$ parts) if

$$
0 \leq n_{1}<\ldots<n_{r} \leq m-1
$$

and

$$
n \equiv n_{1}+\ldots+n_{r}(\bmod m) .
$$

Also, we adopt von Sterneck's notation $(n)_{r}$ to indicate the number of all possible relative decompositions $(\bmod m)$ of $n$ with $r$ parts. The function $(n)_{r}$ can be viewed as an analogue of $p(n, r)$, the number of ordinary partitions of $n$ into $r$ parts. It is easy to see that $p(n, r) \leq(n)_{r}$. For the work on relative decompositions, we refer the readers to [1] and [8]-[12].

From now on, instead of considering $(n)_{r}$, we will focus on restricted relative decompositions $(\bmod m)$ with $r$ parts. More precisely, for each $n=$ $0,1, \ldots, m-1$, let $\mathfrak{G}_{r}(n)$ denote the set of all the relative decompositions $(\bmod m)$ of $n$ with $r$ parts subject to the conditions

$$
n_{i+1}-n_{i} \geq 2 \quad \text { for each } i=1, \ldots, r-1,
$$

and

$$
n_{r}-n_{1} \leq m-2 .
$$

Note that $\mathfrak{G}_{r}(n), n=0,1, \ldots, m-1$, are pairwise disjoint. Also, let $\mathfrak{g}_{r}(n)$ be the cardinality of $\mathfrak{G}_{r}(n)$.

TheOREM 3.1. Let $r$ and $m$ be positive integers with greatest common divisor $d$ and let $j_{1}, j_{2} \in\{0,1, \ldots, m-1\}$. If $d$ divides $j_{1}-j_{2}$ or $j_{1}+j_{2}$, then

$$
\mathfrak{g}_{r}\left(j_{1}\right)=\mathfrak{g}_{r}\left(j_{2}\right) .
$$

In particular, if $r$ and $m$ are relatively prime, then

$$
\mathfrak{g}_{r}(0)=\mathfrak{g}_{r}(1)=\ldots=\mathfrak{g}_{r}(m-1) .
$$

Proof. First, suppose that $d$ divides $j_{1}-j_{2}$. Then we can write $j_{2}=$ $j_{1}+u d$ for some integer $u$. To prove the result, it suffices to find a one-to-one mapping from $\mathfrak{G}_{r}\left(j_{1}\right)$ onto $\mathfrak{G}_{r}\left(j_{2}\right)$. Note that, since $d=(r, m)$, there exist integers $\alpha$ and $\beta$ such that $\alpha r+\beta m=d$.

Next, given an element $\left(n_{1}, \ldots, n_{r}\right)$ in $\mathfrak{G}_{r}\left(j_{1}\right)$, define a new sequence of positive integers

$$
\left(\overline{n_{1}+u \alpha}, \overline{n_{2}+u \alpha}, \ldots, \overline{n_{r}+u \alpha}\right)
$$


where $\bar{z}$ designates the smallest positive residue of $z$ modulo $m$. Finally, denote the sequence $(3.5)$ by $\left(k_{1}, \ldots, k_{r}\right)$, after rearranging the coordinates in a nondecreasing order.

Now, define $\varphi$ from $\mathfrak{G}_{r}\left(j_{1}\right)$ to $\mathfrak{G}_{r}\left(j_{2}\right)$ by assigning to each element $\left(n_{1}, \ldots, n_{r}\right)$ in $\mathfrak{G}_{r}\left(j_{1}\right)$ the sequence $\left(k_{1}, \ldots, k_{r}\right)$ obtained by the procedure described above. The mapping $\varphi$ is clearly one-to-one and onto $(u$ and $\alpha$ are fixed) provided that $\varphi$ is well-defined. Thus, it remains to show that $\left(k_{1}, \ldots, k_{r}\right)$ satisfies $(3.2)-(3.4)$ with $n$ replaced by $j_{2}$. By taking congruences modulo $m$, we find that

$$
\begin{aligned}
\sum_{i=1}^{r} k_{i} & \equiv \sum_{i=1}^{r} n_{i}+u \alpha r \\
& \equiv j_{1}+u d \quad(\text { since } \alpha r+\beta m=d) \\
& \equiv j_{2}(\bmod m)
\end{aligned}
$$

and hence (3.2) is justified.

Next, observe that

$$
\left(k_{1}, \ldots, k_{r}\right)=\left(\overline{n_{1}+u \alpha}, \ldots, \overline{n_{r}+u \alpha}\right) \quad \text { if } \overline{n_{1}+u \alpha} \leq \ldots \leq \overline{n_{r}+u \alpha},
$$

and otherwise,

$$
\left(k_{1}, \ldots, k_{r}\right)=\left(\overline{n_{\nu}+u \alpha}, \ldots, \overline{n_{r}+u \alpha}, \overline{n_{1}+u \alpha}, \ldots, \overline{n_{\nu-1}+u \alpha}\right),
$$

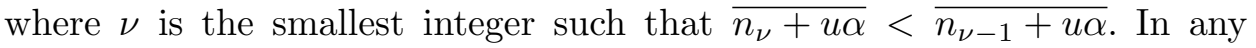
case, (3.3) and (3.4) are satisfied. Therefore, $\mathfrak{G}_{r}\left(j_{1}\right) \cong \mathfrak{G}_{r}\left(j_{2}\right)$ (as sets), i.e., $\mathfrak{g}_{r}\left(j_{1}\right)=\mathfrak{g}_{r}\left(j_{2}\right)$. The case in which $d$ divides $j_{1}+j_{2}$ is proved similarly.

Theorem 3.1 yields immediately the following result.

Corollary 3.2. Given positive integers $r$ and $m$ with $(r, m)=d$, we have

$$
\mathfrak{g}_{r}(l d+k)=\mathfrak{g}_{r}(k)
$$

for any $k \in\{0,1, \ldots, d-1\}$ and any $l \in\{0,1, \ldots, m / d-1\}$.

Now, we are in a position to prove (2.1) and finish the proof of Theorem 2.2 .

COROLlary 3.3. (2.1) holds for any primitive mth root of unity $x$.

Proof. Given $r \in\{1,2, \ldots,[m / 2]\}$, we denote $(r, m)$ by $d$. By the definitions of $\mathcal{C}_{m}(r)$ and $\mathfrak{G}_{r}(j)$,

$$
\mathcal{C}_{m}(r)=\mathfrak{G}_{r}(0) \cup \mathfrak{G}_{r}(1) \cup \ldots \cup \mathfrak{G}_{r}(m-1) .
$$


Then, by (3.6), the disjointness of the sets $\mathfrak{G}_{r}(j)$, and Corollary 3.2,

$$
\begin{aligned}
\sum_{\vec{v} \in \mathcal{C}_{m}(r)} x^{n_{1}+\ldots+n_{r}} & =\sum_{j=0}^{m-1} \mathfrak{g}_{r}(j) x^{j}=\sum_{k=0}^{d-1} \sum_{l=0}^{m / d-1} \mathfrak{g}_{r}(l d+k) x^{l d+k} \\
& =\sum_{k=0}^{d-1} \mathfrak{g}_{r}(k) x^{k} \sum_{l=0}^{m / d-1} x^{l d}=0
\end{aligned}
$$

where the last equality follows from the fact that $x^{d}$ is a primitive $(m / d)$ th root of unity. Hence, (2.1) is established, and so is Theorem 2.2.

\section{Proof of (1.6)}

Lemma 4.1 (Worpitzky's theorem). Let $\mathbb{K}\left(a_{n} / 1\right)$ be the continued fraction defined in (1.1). If $\left|a_{n}\right| \leq 1 / 4$, then $\mathbb{K}\left(a_{n} / 1\right)$ converges. Moreover, all approximants $P_{n} / Q_{n}$ defined in (1.2) are in the disk $|w|<1 / 2$, and the value of the continued fraction is in the disk $|w| \leq 1 / 2$.

Proof. See [3, p. 35].

Definition. Let $\Lambda$ be a set of functions, all defined on the same domain $G$, and suppose that for every compact subset $F \subset G$ there is a number $M(F)>0$ such that

$$
|f(z)| \leq M(F)
$$

for all $f \in \Lambda$ and $z \in F$. Then $\Lambda$ is said to be uniformly bounded inside $G$.

Lemma 4.2 (Vitali's theorem). Let $G$ be a domain and let $\left\{f_{n}\right\}$ be a sequence of analytic functions in $G$. Suppose that $\left\{f_{n}\right\}$ is uniformly bounded inside $G$ and converges on a set of points $E \subset G$ with a limit point in $G$. Then $\left\{f_{n}\right\}$ converges uniformly inside $G$.

Proof. See [4, Vol. I, pp. 415-417].

LEMMA 4.3. For each fixed primitive root of unity $x, R(a)$ is an analytic function of a inside the domain $G=\{a:|a|<1 / 4\}$.

Proof. For convenience, denote $P_{n}(a) / Q_{n}(a)$ by $f_{n}(a)$ for each $n \in \mathbb{N}$. By Lemma 4.1, we have

$$
\left|f_{n}(a)\right|<\frac{1}{2}, \quad \text { for each } n \in \mathbb{N} \text { and each } a \in G,
$$

and $\left\{f_{n}(a)\right\}$ converges to $R(a)$ in the domain $G$. Hence, $\left\{f_{n}\right\}$ is uniformly bounded inside $G$. On the other hand, by Lemma 2.1, $P_{n}(a)$ and $Q_{n}(a)$ are polynomials in $a$ with coefficients in $\mathbb{C}$. This, combined with (4.1), implies that $\left\{f_{n}\right\}$ is indeed a sequence of analytic functions in $G$. Therefore, by Lemma $4.2,\left\{f_{n}(\cdot)\right\}$ converges uniformly to $R(\cdot)$ in $G$. Finally, the analyticity of $R(\cdot)$ follows from Weierstrass's uniform convergence theorem [4, p. 333]. 
We should mention that Ramanujan recorded (1.6) with no indication of any admissible range for $a$. However, this can be done without too much difficulty. Indeed, the domain $G$ in Lemma 4.3 is, in general, the best possible circular domain for $a$ according to Lemma 4.1 and the fact that the continued fraction $\mathbb{K}(a / 1)$ diverges for real $a$ with $a<-1 / 4$. In the following, we restate (1.6) in a more precise way.

THEOREM 4.4. Let $x$ be a primitive $m$ th root of unity and $|a|<1 / 4$. Let $R(a)$ be the continued fraction defined in (1.5). Then

$$
R(a)=\frac{P_{m-1}(a)+\frac{1}{2}\left\{-1+\sqrt{1+4 a^{m}}\right\}}{Q_{m-1}(a)},
$$

where

$$
\frac{P_{m-1}(a)}{Q_{m-1}(a)}=\frac{a}{1}+\frac{a x}{1}+\ldots+\frac{a x^{m-2}}{1} .
$$

Proof. Observe that $R(a)$ becomes a periodic continued fraction when $x^{m}=1$. Hence,

$$
\begin{aligned}
R(a) & =\frac{a}{1}+\frac{a x}{1}+\frac{a x^{2}}{1}+\ldots+\frac{a x^{m-1}}{1}+\frac{R(a)}{1} \\
& =\frac{P_{m}(a)+R(a) P_{m-1}(a)}{Q_{m}(a)+R(a) Q_{m-1}(a)}
\end{aligned}
$$

by (1.3). The identity (4.2) gives a quadratic equation in $R(a)$, i.e.,

$$
Q_{m-1}(a) R(a)^{2}-\left\{P_{m-1}(a)-Q_{m}(a)\right\} R(a)-P_{m}(a)=0 .
$$

Solving (4.3) by the quadratic formula, we obtain

$$
R(a)=\frac{\left\{P_{m-1}(a)-Q_{m}(a)\right\} \pm \sqrt{\left\{P_{m-1}(a)-Q_{m}(a)\right\}^{2}+4 P_{m}(a) Q_{m-1}(a)}}{2 Q_{m-1}(a)} .
$$

By Theorem 2.2, the last identity can be rewritten as

$$
R(a)=\frac{P_{m-1}(a)+\frac{1}{2}\left\{-1 \pm \sqrt{1+4\left\{P_{m}(a) Q_{m-1}(a)-P_{m-1}(a) Q_{m}(a)\right\}}\right\}}{Q_{m-1}(a)} .
$$

Let us write $x=\exp (2 \pi i h / m)$, with $(h, m)=1$. Then, by (1.4),

$$
\begin{aligned}
P_{m}(a) Q_{m-1}(a) & -P_{m-1}(a) Q_{m}(a) \\
= & (-1)^{m-1} a \cdot a x \cdot \ldots \cdot a x^{m-1}=(-1)^{m-1} a^{m} x^{m(m-1) / 2} \\
= & (-1)^{m-1} a^{m} e^{\frac{2 \pi i h}{m} \cdot \frac{m(m-1)}{2}}=(-1)^{(h+1)(m-1)} a^{m}=a^{m},
\end{aligned}
$$

where the last equality follows from the fact that $h$ and $m$ are coprime. 
Hence, from (4.4),

$$
R(a)=\frac{P_{m-1}(a)+\frac{1}{2}\left\{-1+\sqrt{1+4 a^{m}}\right\}}{Q_{m-1}(a)}
$$

or

$$
R(a)=\frac{P_{m-1}(a)+\frac{1}{2}\left\{-1-\sqrt{1+4 a^{m}}\right\}}{Q_{m-1}(a)} .
$$

Now, it remains to exclude (4.6). By Lemma 2.1, $P_{m-1}(a)$ and $Q_{m-1}(a)$ are both polynomials in $a$ and approach 0 and 1, respectively, when $a$ tends to zero. Hence, when $a$ is inside a small neighborhood of 0 , the quantity on the right side of (4.6) will be outside the disk $|w| \leq 1 / 2$, which contradicts Lemma 4.1. This implies that (4.5) is valid for $|a| \leq \varrho$, where $\varrho$ is a small positive number depending on $m$ only. Finally, the desired result follows by Lemma 4.3 and analytic continuation.

To conclude this section, we state Schur's result on the case $a=1$ and relate it to Theorem 4.4 .

TheOREM 4.5 ([7, pp. 319-321]). Let $F(x):=R(1)$, where $x$ is a primitive mth root of unity. If $m$ is a multiple of $5, F(x)$ diverges. Otherwise, $F(x)$ converges and

$$
F(x)=\alpha F(\alpha) x^{(\alpha \varrho m-1) / 5},
$$

where $\alpha$ denotes the Legendre symbol $\left(\frac{m}{5}\right)$ and $\varrho$ is the least positive residue of $m$ modulo 5. Moreover, in the latter case,

$$
P_{m-1}(1)=\frac{1}{2}(1-\alpha) \quad \text { and } \quad Q_{m-1}(1)=\alpha x^{(1-\alpha \varrho m) / 5} .
$$

According to the table on page 57 of his lost notebook, Ramanujan apparently tried to establish results like (4.8) to obtain (4.7). Unfortunately, this table is not completely correct by comparison to that of Schur [7, p. 319], and probably led Ramanujan to the following (incorrect) result which was recorded on page 383 of his second notebook [6].

"If $u:=x^{1 / 5} F(x)$, then $u^{2}+u-1=0$ when $x^{n}=1$, where $n$ is any positive integer except multiples of 5 in which case $u$ is not definite."

To obtain the result above, Ramanujan might have used his table and applied Theorem 4.4 with $a=1$. If so, Ramanujan intended Theorem 4.4 to be valid for $a=1$. Indeed, this turns out to be the case since, when $a=1$, Theorem 4.4 reduces to (4.7) simply by using (4.8). Therefore, it is likely that Theorem 4.4 holds for a larger region of $a$. Finally, we emphasize that the convergence problem of $F(x)$ on the unit circle except primitive roots of unity is still unsolved. 
5. A generalization of Theorem 4.4. On page 57 of his lost notebook, Ramanujan generalizes Theorem 4.4 by considering the continued fraction

$$
\varepsilon=\frac{1}{1}+\frac{a x}{1}+\frac{a x^{2}}{1}+\ldots+\frac{a x^{n}}{1+\lambda \varepsilon} .
$$

Observe that, by choosing $\lambda=a$, the continued fraction (5.1) reduces to $a^{-1} R(a)$.

Let, for $j \in \mathbb{N}$,

$$
(x)_{j}:=(1-x)\left(1-x^{2}\right) \ldots\left(1-x^{j}\right),
$$

and define the Gaussian coefficients by

$$
\left[\begin{array}{l}
k \\
0
\end{array}\right]_{x}=\left[\begin{array}{l}
k \\
k
\end{array}\right]_{x}=1
$$

and by

$$
\left[\begin{array}{c}
k \\
l
\end{array}\right]_{x}=\frac{(x)_{k}}{(x)_{l}(x)_{k-l}}
$$

when $0<l<k$. Here we only consider integral values for $k$ and $l$. Note that (5.2) is indeed a polynomial in $x$.

THEOREM 5.1. Let

$$
A_{n}(a)=\sum_{j=0}^{[(n+1) / 2]} a^{j} x^{j^{2}}\left[\begin{array}{c}
n-j+1 \\
j
\end{array}\right]_{x}, \quad \text { for } n \in \mathbb{N},
$$

$A_{0} \equiv 1, A_{-1} \equiv 1$, and $A_{-2} \equiv 0$. Then, for $n \geq 0$,

$$
\text { (iv) } \frac{1}{1}+\frac{a x}{1}+\frac{a x^{2}}{1}+\ldots+\frac{a x^{n}}{1+\eta}=\frac{A_{n-1}(a x)+\eta A_{n-2}(a x)}{A_{n}(a)+\eta A_{n-1}(a)} \text {. }
$$

Proof. Observe that $A_{n}(a)$ is indeed the numerator of the $n$th convergent of the continued fraction $a / R(a)$, which is a result due to Ramanujan and can be found in Berndt's book [2, p. 31, Entry 16]. In other words,

$$
\frac{A_{n}(a)}{B_{n}(a)}=1+\frac{a x}{1}+\frac{a x^{2}}{1}+\ldots+\frac{a x^{n}}{1},
$$

where $B_{n}(a)$ denotes the corresponding denominator. In fact, one can easily show that $B_{n}(a)=a^{-1} P_{n+1}(a)$. Furthermore,

$$
\frac{A_{n}(a)}{B_{n}(a)}=1+\frac{a x}{1+\frac{a x^{2}}{1}+\frac{a x^{3}}{1}+\ldots+\frac{a x^{n}}{1}}
$$




$$
\begin{aligned}
& =1+\frac{a x}{A_{n-1}(a x) / B_{n-1}(a x)} \\
& =\frac{A_{n-1}(a x)+a x B_{n-1}(a x)}{A_{n-1}(a x)} .
\end{aligned}
$$

The equality (5.3) implies immediately that

$$
B_{n}(a)=A_{n-1}(a x)
$$

and

$$
A_{n}(a)=A_{n-1}(a x)+a x A_{n-2}\left(a x^{2}\right),
$$

which proves (ii). By (5.4), (i) follows from (1.4). (iii) is simply the first recurrence relation in (1.3). Finally, (iv) follows from (1.3) and (5.4).

Theorem 5.2. Let $|a|<1 / 4,|\lambda|<1 / 4$, and $|x| \leq 1$. If $\varepsilon$ is the continued fraction defined in (5.1), then

$$
\varepsilon=\frac{A_{n-2}(a x)+Z}{A_{n-1}(a)},
$$

where

$$
\lambda Z^{2}+\left\{A_{n}(a)+\lambda A_{n-2}(a x)\right\} Z=(-a)^{n} x^{n(n+1) / 2},
$$

and the ambiguous sign in the solution of (5.5) is always positive.

Proof. Throughout the proof, we restrict $a, \lambda$, and $x$ to be inside the prescribed areas. First, the convergence of the continued fraction $\varepsilon$ is guaranteed by Lemma 4.1. By Theorem 5.1(iv),

$$
\varepsilon=\frac{A_{n-1}(a x)+\lambda \varepsilon A_{n-2}(a x)}{A_{n}(a)+\lambda \varepsilon A_{n-1}(a)} .
$$

Solving the quadratic equation deduced from (5.6), we have

$$
\varepsilon=\frac{2 \lambda A_{n-2}(a x)-Y \pm \sqrt{Y^{2}+4 \lambda(-a)^{n} x^{n(n+1) / 2}}}{2 \lambda A_{n-1}(a)},
$$

where we have used Theorem 5.1(i) and, for convenience, we denote $\lambda A_{n-2}(a x)+A_{n}(a)$ by $Y$. Hence,

$$
\varepsilon=\frac{A_{n-2}(a x)+Z}{A_{n-1}(a)},
$$

where

$$
Z=\frac{1}{2 \lambda}\left[-Y \pm \sqrt{Y^{2}+4 \lambda(-a)^{n} x^{n(n+1) / 2}}\right] .
$$

One can easily check that $Z$ satisfies the equation (5.5). Therefore, it remains to verify that the ambiguous sign is always positive. By Lemma 2.1, both 
$A_{n}(a)$ and $A_{n-2}(a x)$ are polynomials in $a$ and approach 1 when $a$ tends to 0 , and hence

$$
Y \rightarrow 1+\lambda \quad \text { as } a \rightarrow 0 .
$$

Now, let us first fix $x$ and $\lambda$, with $0<\lambda<1 / 4$. Then, by (5.7)-(5.9), when $a$ tends to $0, \varepsilon$ approaches 1 and $-1 / \lambda$, respectively, according to the "+" and "-" signs in (5.8). However, by Lemma 4.1, $\varepsilon$ converges to a value in the disk $|w| \leq 2$, which excludes the value $-1 / \lambda$ when $a$ is small enough. In other words, the "+" sign is always correct when $a$ is in a small neighborhood of the origin. Furthermore, a similar argument used in the proof of Lemma 4.3 shows that $\varepsilon$ is an analytic function of $a$. Therefore, by analytic continuation, Theorem 5.2 is valid for $|x| \leq 1,|a|<1 / 4$, and $0<\lambda<1 / 4$. Finally, the desired domain for $\lambda$ can be obtained by analytic continuation since $\varepsilon$ is also analytic in $\lambda$.

In addition to Theorems 5.1 and 5.2, Ramanujan recorded the following two results on p. 57 of his lost notebook [5].

$$
\text { If } x^{n}=1 \text { ( } x \text { primitive), then } A_{n-1}(a)+a A_{n-3}(a x)=1 \text {, }
$$

$$
A_{n-2}(a) A(a x)-A_{n-3}(a x) A(a)=(-a)^{n-1} x^{n(n-1) / 2} A\left(a x^{n}\right) .
$$

Pro of of (5.10). This result is actually a restatement of Theorem 2.2.

Pr o of of (5.11). It is clear that Ramanujan uses $A(a)$ as the limit of $A_{n}(a)$ when $n$ tends to infinity without any indication. It is easily seen that, by a similar argument, Theorem 5.1(ii) remains valid if we replace $A_{n}(\cdot)$ by $A(\cdot)$. In other words,

$$
A(a)=A(a x)+a x A\left(a x^{2}\right) .
$$

By Theorem 5.1(ii) and (5.12),

$$
\begin{aligned}
& A_{n-2}(a) A(a x)-A_{n-3}(a x) A(a) \\
= & \left\{A_{n-3}(a x)+a x A_{n-4}\left(a x^{2}\right)\right\} A(a x)-A_{n-3}(a x)\left\{A(a x)+a x A\left(a x^{2}\right)\right\} \\
= & -a x\left\{A_{n-3}(a x) A\left(a x^{2}\right)-A_{n-4}\left(a x^{2}\right) A(a x)\right\} .
\end{aligned}
$$

Note that the expression inside the parentheses on the far right side of (5.13) is exactly the expression on left side with the subscripts reduced by 1 and with $a$ replaced by $a x$. Hence, we can reiterate the recurrence above to obtain

$$
\begin{aligned}
& A_{n-2}(a) A(a x)-A_{n-3}(a x) A(a) \\
& \quad=(-a x)\left(-a x^{2}\right) \ldots\left(-a x^{n-1}\right)\left\{A_{-1}\left(a x^{n-1}\right) A\left(a x^{n}\right)-A_{-2}\left(a x^{n}\right) A\left(a x^{n-1}\right)\right\} \\
& \quad=(-a)^{n-1} x^{n(n-1) / 2} A\left(a x^{n}\right),
\end{aligned}
$$

since $A_{-1} \equiv 1$ and $A_{-2} \equiv 0$. 
Re m a r k. Because of the appearance of (5.10) in the lost notebook, it is very likely that the proof of Theorem 4.4 we gave in Section 4 is essentially the one that Ramanujan had. However, we have no clue how Ramanujan found and proved Theorem 2.2.

Acknowledgments. The author would like to thank Professor B. Berndt for bringing this problem to his attention and for many valuable suggestions. The author is very grateful to Professor A. Schinzel, the Editor of Acta Arithmetica, for providing information on Stern's and von Sterneck's work on relative decompositions.

\section{References}

[1] P. Bachmann, Niedere Zahlentheorie, New York, 1991.

[2] B. Berndt, Ramanujan's Notebooks, Part III, Springer, New York, 1991.

[3] L. Lorentzen and H. W aadeland, Continued Fractions with Applications, NorthHolland, Amsterdam, 1992.

[4] A. I. Markushevich, Theory of Functions of a Complex Variable, English translation by R. A. Silverman, Chelsea, New York, 1985.

[5] S. Ramanujan, The Lost Notebook and Other Unpublished Papers, Narosa, New Delhi, 1988.

[6] -, Notebooks (2 volumes), Tata Institute of Fundamental Research, Bombay, 1957.

[7] I. Schur, Ein Beitrag zur additiven Zahlentheorie und zur Theorie der Kettenbrüche, S.-B. Preuss. Akad. Wiss. Phys.-Math. Kl. (1917), 302-321.

[8] H. Stern, Ueber eine der Theilung der Zahlen ähnliche Untersuchung und deren Anwendung auf die Theorie der quadratischen Reste, J. Reine Angew. Math. 61 (1863), 66-94.

[9] - Zur Theorie der quadratischen Reste, ibid., 334-349.

[10] R. Daublebsky v. Sterneck, Ein Analogon zur additiven Zahlentheorie, Sitzungsber. Wiener Akad. 111 (1902), 1567-1601.

[11] —, Ein Analogon zur additiven Zahlentheorie, ibid. 113 (1904), 326-340.

[12] —, Über die Kombinationen der Potenzreste einer Primzahl zu bestimmten Summen, ibid. 114 (1905), 711-758.

Department of Mathematics

University of Illinois

1409 West Green Street

Urbana, Illinois 61801

U.S.A.

E-mail: shuang@math.uiuc.edu 